\title{
Туннельный ток во встречных диодах Шоттки, образованных контактами между вырожденным GaN n-типа и металлом
}

\author{
() И.О. Майборода, Ю.В. Грищенко, И.С. Езубченко, И.С. Соколов, И.А. Черных, \\ А.А. Андреев, М.Л. Занавескин
}

Национальный исследовательский центр „Курчатовский институт“, 123182 Москва, Россия

E-mail: mrlbr@mail.ru

(Получена 4 июля 2017 г. Принята к печати 9 августа 2017 г.)

\begin{abstract}
Проведено исследование нелинейного поведения зависимости тока от напряжения в симметричных контактах между металлом и вырожденным $\mathrm{GaN} n$-типа, образующих встречные диоды Шоттки при концентрациях свободных носителей в $\mathrm{GaN}$ от $1.5 \cdot 10^{19}$ до $2.0 \cdot 10^{20} \mathrm{~cm}^{-3}$. Показано, что при концентрации электронов $2.0 \cdot 10^{20} \mathrm{~cm}^{-3}$ проводимость между металлом (хромом) и $\mathrm{GaN}$ осуществляется путем туннелирования электронов, а удельное сопротивление контакта $\mathrm{Cr}-\mathrm{GaN}$ при этом составляет $0.05 \mathrm{OM} \cdot$ мм. Разработан метод определения параметров потенциальных барьеров по вольт-амперным характеристикам симметричных встречных контактов. Учтено влияние сильной неоднородности распределения плотности тока и напряжения по площади контактов при малом удельном сопротивлении контактов. Для контактов $\mathrm{Cr}-n^{+}-\mathrm{GaN}$ получено значение высоты потенциального барьера, равное $0.47 \pm 0.04$ эВ.
\end{abstract}

DOI: $10.21883 / F T P .2018 .06 .45928 .8568$

\section{1. Введение}

Транзисторы с высокой подвижностью электронов на основе широкозонных нитридных полупроводников $\mathrm{GaN}, \mathrm{AlN}$ и $\mathrm{AlGaN}$ (GaN HEMT) являются важными компонентами современной микроэлектроники [1]. Одним из перспективных подходов формирования омических контактов для GaN HEMT с низким сопротивлением является формирование так называемых доращиваемых (или невплавных) контактов, создаваемых на основе $\mathrm{GaN}$ с высоким содержанием легирующей примеси $n$-типа $\left(n^{+}-\mathrm{GaN}\right)[2,3]$. Высокая концентрация электронов в $n^{+}$-GaN обеспечивает низкое удельное сопротивление интерфейса металл-полупроводник (М-ПП), что позволяет добиться удельного сопротивления интерфейса ниже $10^{-6} \mathrm{OM} \cdot \mathrm{cm}^{2}$ и сопротивления контактов менее 0.1 Ом на миллиметр ширины контакта [4].

Сопротивление контакта во многом определяется механизом переноса электронов через интерфейс металл-полупроводник. В контактах к нитриду галлия на границе металл-GaN обычно возникает обедненный слой, который выступает в качестве потенциального барьера для электронов (барьер Шоттки). В зависимости от высоты и ширины барьера транспорт заряда через контакт может осуществляться за счет термоэлектронной, термополевой или полевой (туннелирование) эмиссии электронов [5,6]. При концентрациях свободных электронов более $10^{20} \mathrm{~cm}^{-3}$ глубина обедненного слоя (т. е. толщина потенциального барьера) на границе М-ПП становится достаточно малой, чтобы транспорт электронов осуществлялся за счет туннелирования. При туннельном механизме прохождения электронов через барьер Шоттки в контакте М-ПП сопротивление интерфейса достаточно мало, чтобы использовать такой контакт в качестве омического. Все перечисленные механизмы проводимости являются нелинейными.
Изучение нелинейного поведения контактов является мощным инструментом для получения информации о характеристиках перехода $\mathrm{M}-П П$, знание которой необходимо для управления сопротивлением контактов. Для измерения вольт-амперных характеристик (BAX) переходов М-ПП обычно используется асимметричная схема из двух различных контактов: контакта, содержащего исследуемый переход М-ПП, и омического контакта с полупроводником. Второй контакт в этой схеме должен иметь малое сопротивление по сравнению с исследуемым переходом. Асимметрия сопротивлений необходима для того, чтобы влиянием омического контакта на BAX исследуемого перехода можно было пренебречь. Асимметричные схемы успешно применяются для изучения переходов с относительно высоким удельным сопротивлением (обычно более $10^{-4} \mathrm{OM} \cdot \mathrm{cm}^{2}$ ), в которых проводимость возникает благодаря термополевой и термоэлектронной эмиссии [5,7-9].

Для исследования интерфейсов $\mathrm{M}-$ ПП с низким удельным сопротивлением стандартные методы изучения нелинейности оказываются неприменимы. Это связано с двумя причинами. Первая - исследуемый переход сам по себе имеет малое сопротивление, из-за чего изготовить омический контакт, сопротивление которого пренебрежимо мало по сравнению с исследуемым переходом, в большинстве случаев оказывается технологически невозможным. Вторая причина состоит в том, что из-за неравномерного растекания тока различные участки контакта находятся под разным напряжением, и через них соответственно протекает ток с различной плотностью. Это не позволяет получить из экспериментальной ВАХ непосредственную зависимость плотности тока от напряжения. Ввиду перечисленных проблем точное аналитическое описание BAX систем планарных контактов с малым сопротивлением оказывается слож- 
ной нелинейной задачей, что является причиной отсутствия методов анализа нелинейного поведения подобных контактов.

В данной работе решались две задачи: первая разработка простого и удобного для практического использования метода анализа нелинейного поведения BAX планарных контактов металл-полупроводник с низким удельным сопротивлением; вторая - экспериментальное исследование туннельных контактов между металлом и вырожденным GaN n-типа. Статья организована следующим образом. В разд. 2 даны основные определения и выражения, необходимые для описания неравномерного растекания тока в планарных контактах. В разд. 3 приведены детали эксперимента по изготовлению и измерению характеристик контактов хром $(\mathrm{Cr})-n^{+}-\mathrm{GaN}$ при концентрациях свободных носителей в $\mathrm{GaN}$ от $1.5 \cdot 10^{19}$ до $2.0 \cdot 10^{20} \mathrm{~cm}^{-3}$ и показано, что при концентрации электронов $2.0 \cdot 10^{20} \mathrm{~cm}^{-3}$ проводимость осуществляется за счет полевой эмиссии. В разд. 4 вводится модель туннельного контакта между металлом и вырожденным полупроводником. В разд. 5 приведен вывод аналитического выражения для описания ВАХ системы из двух нелинейных контактов, соединенных последовательно навстречу друг другу. В разд. 6 полученное выражение использовано для определения высоты и ширины потенциального барьера на границе $\mathrm{Cr}-\mathrm{GaN}$ в экспериментальных образцах. Статья завершается кратким заключением, обобщающим основные результаты работы.

\section{2. Неравномерное растекание тока в планарных контактах}

Рассмотрим планарный контакт между металлом и полупроводниковой пленкой, толщина которой мала по сравнению с размерами контактной площадки (рис. 1), а слоевое сопротивление полупроводника, напротив, много больше слоевого сопротивления металла. Полное падение напряжения на контакте $U_{c}$, измеряемое экспериментально, будет равно разности между потенциалом на поверхности металла и потенциалом в полупроводнике у переднего края контакта. Сопротивлением контакта $R_{c}$ называется значение производной напряжения $U_{c}$ по току при $U_{c}$, равном нулю [7], т. е.

$$
R_{c}=\left[\frac{d U_{c}}{d I}\right]_{U_{c}=0} .
$$

Таким образом, при линейной зависимости тока от напряжения, сопротивление соответствует обратному углу наклона ВАХ, при нелинейном характере зависимости сопротивление определяется по углу наклона касательной к BAX при напряжении и токе, равных нулю.

Неравномерное растекание тока приводит к тому, что напряжение на интерфейсе $\mathrm{M}-$ ПП меняется с увеличением расстояния $x$ от края контакта по закону

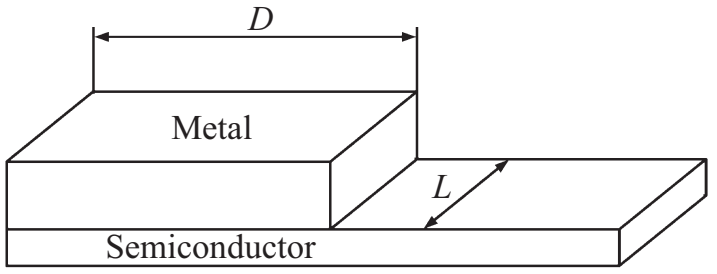

Рис. 1. Схематичное изображение планарного контакта металл-полупроводник: $L-$ ширина контакта, $D-$ длина контакта.

$U(x)=U_{c} \cosh \left[(D-x) / \sqrt{\sigma-R_{s}}\right] / \sinh \left(D / \sqrt{\sigma / R_{s}}\right) \quad$ [7], где $\sigma-$ сопротивление единицы площади интерфейса металл-полупроводник, $R_{s}-$ слоевое сопротивление полупроводника, $D$ - длина контакта, a $D_{0}=\sqrt{\sigma-R_{s}}-$ эффективная длина контакта (transfer length [7]), которая равна расстоянию от границы, на котором плотность тока падает в $e$ раз. При малых $\sigma$ и $R_{s}$ длина контакта $D \gg D_{0}$, что позволяет считать контакт полубесконечным и использовать выражение

$$
U(x)=U_{c} \exp \left(-x / D_{0}\right)
$$

При этом сопротивление всего контакта равно $R_{c}=\sqrt{R_{s} \sigma} / L$, где $L-$ ширина контакта, следовательно,

$$
\sigma=\left(L R_{c}\right)^{2} / R_{s}
$$

Получается, что при уменьшении сопротивления интерфейса М-ПП уменьшается эффективная длина контакта $D_{0}$. Для качественных омических контактов $D_{0}$ составляет порядка единиц микрон, и условие $D \gg D_{0}$ выполняется практически всегда. Таким образом, в низкоомных контактах величина напряжения на интерфейсе М-ПП существенно меняется по площади контакта, и полный ток через контакт должен вычисляться как интеграл плотности тока по площади всего интерфейса:

$$
I\left(U_{c}\right)=L \int_{0}^{\infty} j\left[U\left(U_{c}, x\right)\right] d x
$$

\section{3. Эксперимент}

Для экспериментального исследования нелинейного поведения BAX контактов М-ПП была изготовлена серия образцов пленок $\mathrm{GaN}$ с различной концентрацией кремния. Легированные кремнием слои $\mathrm{GaN}$ толщиной 200 нм были выращены методом аммиачной молекулярно-лучевой эпитаксии [3] на темплейтах $\mathrm{GaN}$ на сапфире при температуре $850^{\circ} \mathrm{C}$. Концентрация носителей заряда $n_{0}$ в пленках $\mathrm{GaN}: \mathrm{Si}$ определялась четырехзондовым методом Ван-дер-Пау. На полученных пленках были сформированы тестовые структуры типа „длинная линия“ TLM (transmission line method) с зазорами 5, 10, 

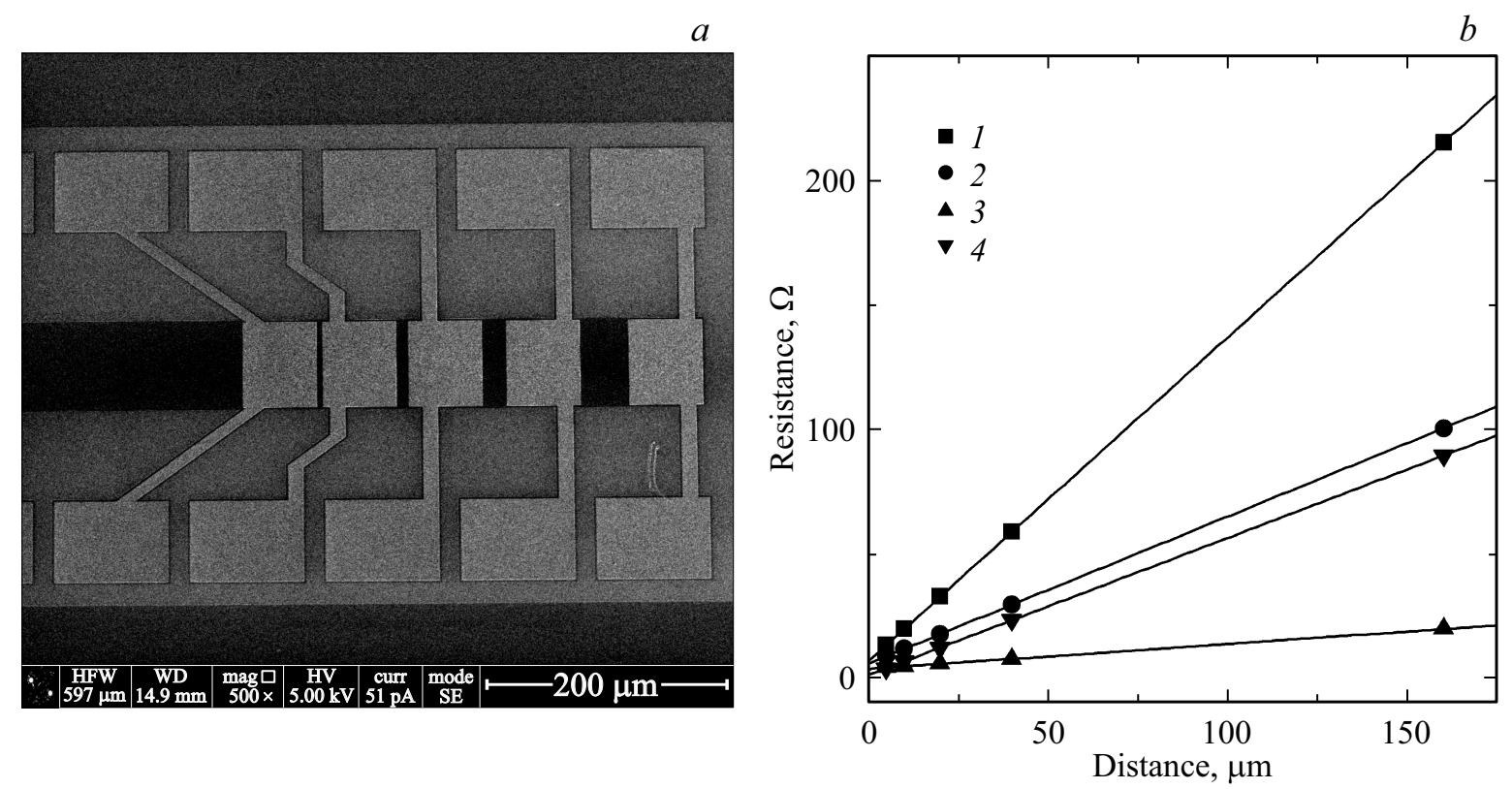

Рис. 2. $a-$ изображение ТLМ-модуля с зазорами $5,10,20,40$ и 160 мкм, снятое с помощью сканирующего электронного микроскопа; $b$ - зависимость сопротивлений от расстояния между контактными площадками модулей TLM на образцах $1-4$.

20, 40 и 160 мкм, шириной 100 мкм (рис. 2, a). Металлические контактные площадки создавались путем обратной фотолитографии и последующим электронно-лучевым напылением металлов $\mathrm{Cr}(0.03$ мкм)/Au (0.2 мкм) поверх $\mathrm{GaN}: \mathrm{Si}$. Ширина и длина контактных площадок составляли 100 и 50 мкм соответственно. Измерения токов и напряжений в тестовых модулях осуществлялись с помощью источника-измерителя KEITHLEY 2636В и зондовой установки Cascade Microtech PM5 четырехзондовым методом. Зависимости сопротивления модулей в TLM-схемах от расстояния между контактными площадками для образцов $\mathrm{GaN}: \mathrm{Si}$ с разной концентрацией кремния приведены на рис. $2, b$. Рассчитанные по результатам TLM-измерений значения удельного сопротивления омических контактов $(\rho)$ и слоевого сопротивления $n^{+}-\mathrm{GaN}\left(R_{s}\right)$, а также значения удельного сопротивления интерфейса $\mathrm{M}-П П(\sigma)$ и эффективной длины контакта $\left(D_{0}\right)$, рассчитанные с помощью формул из предыдущего раздела, приведены в таблице.

BAX измерялись на контактах TLM-модуля с зазором 10 мкм. Падение напряжения $V$ на контактах $\mathrm{Cr}-\mathrm{GaN}: \mathrm{Si}$ вычислялось по формуле $V=\left(V_{\text {total }}-I R_{s} S / L\right)$, где $I-$

Рассчитанные параметры исследованных структур

\begin{tabular}{c|c|r|c|c|c}
\hline $\begin{array}{c}\text { № } \\
\text { образца }\end{array}$ & $\begin{array}{c}n_{0}, \\
10^{19} \mathrm{~cm}^{-3}\end{array}$ & $\begin{array}{c}R_{s}, \\
\text { Ом }\end{array}$ & $\begin{array}{c}\sigma, \\
\text { Ом·мм }\end{array}$ & $\begin{array}{c}\sigma, \\
10^{-6} \mathrm{OM} \cdot \mathrm{cm}^{2}\end{array}$ & $\begin{array}{c}D_{0}, \\
\text { мкм }\end{array}$ \\
\hline 1 & 1.5 & 130 & 0.32 & 8.1 & 2.5 \\
2 & 4.6 & 59 & 0.28 & 13.0 & 4.7 \\
3 & 12 & 10 & 0.17 & 28.9 & 17 \\
4 & 20 & 55 & 0.05 & 0.45 & 0.9
\end{tabular}

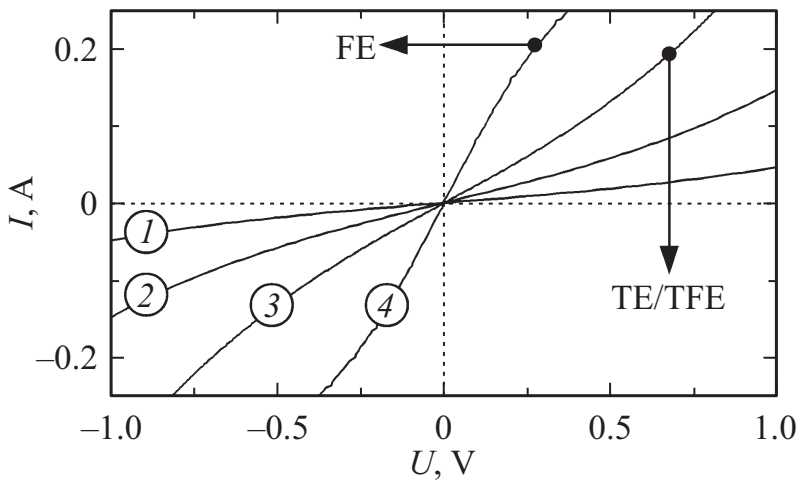

Рис. 3. ВАХ тестовых модулей с зазорами 10 мкм на образцах $1-4$.

ток, $V_{\text {total }}$ - полное падение напряжения на измерительном модуле, $S$ - длина зазора между контактами, $L$ - ширина контакта. Полученные зависимости $I(V)$ приведены на рис. 3.

Как видно на рис. 3 и из таблицы, проводимость контактов растет с увеличением концентрации носителей в $n^{+}$-GaN. При малых токах BAX полученных контактов близки к линейным. При напряжениях более 0.2 В все BAX имеют явно нелинейный характер. На образцах 1-3 производная тока по напряжению растет с увеличением напряжения, что характерно для контактов, в которых проводимость реализуется за счет термоэмиссии и термополевой эмиссии [7-10].

Для полевой эмиссии, напротив, характерно уменьшение дифференциального сопротивления с увеличением напряжения, поскольку толщина барьера на границе 
металл-полупроводник зависит от величины приложенного напряжения и направления тока (здесь и далее все выражения приведены в системе СГС):

$$
s=\sqrt{\frac{\varepsilon(U+\varphi / e)}{2 \pi e n_{0}}},
$$

где $s$ - толщина барьера, $\varepsilon$ - диэлектрическая проницаемость материала, $U$ - падение напряжения на барьере, $e-$ заряд электрона, $\varphi$ - высота потенциального барьера, $n_{0}$ - концентрация свободных носителей в полупроводнике. Измерительные модули представляют собой два диода Шоттки, включенные навстречу друг другу. Как следует из выражения (4), при приложении внешнего напряжения толщина барьера на одном из контактов увеличивается, что приводит к увеличению его сопротивления и к увеличению сопротивления всей цепи.

Таким образом, увеличение дифференциального сопротивления на образце 4 с концентрацией носителей $2 \cdot 10^{20} \mathrm{~cm}^{-3}$ показывает, что проводимость контактов на нем осуществляется за счет полевой эмиссии. О том же говорит и численная оценка с помощью выражения (4), при $\varphi$ менее 1 эВ и $n_{0}=2 \cdot 10^{20} \mathrm{~cm}^{-3}$ значение толщины $s_{0}$ будет составлять не более $1.9 \mathrm{HM}$, в то время как для первого образца $s_{0} \approx 8 \mathrm{HM}$, что исключает туннелирование.

\section{4. Модель контакта между металлом и вырожденным полупроводником}

При концентрации электронов более $10^{20} \mathrm{~cm}^{-3} \mathrm{GaN}$ является вырожденным полупроводником, в котором уровень Ферми находится выше дна зоны проводимости. Это дает возможность описывать туннельный переход на границе металл- $\mathrm{GaN}$ как туннельный переход металлметалл. При малых напряжениях ВАХ такого перехода описывается следующим выражением [11]:

$$
\begin{aligned}
j(V)= & \left(\frac{e}{2 \pi h s_{0}^{2}}\right)\left\{(\varphi-e U / 2) \exp \left[-A(\varphi-e U / 2)^{1 / 2}\right]\right. \\
& \left.-(\varphi+e U / 2) \exp \left[-A(\varphi+e U / 2)^{1 / 2}\right]\right\}
\end{aligned}
$$

где $A=4 \pi s\left(2 m^{*}\right)^{1 / 2} / h, j-$ плотность тока, $m^{*}-$ эффективная масса электрона в полупроводнике, $h-$ постоянная Планка, $\varphi$ - средняя высота потенциального барьера относительно уровня Ферми, $e-$ заряд электрона, $s$ - толщина барьера, $U-$ приложенная разность потенциалов.

Чтобы описать контакт М-ПП с помощью выражения (5), нужно учесть, что толщина потенциального барьера меняется с увеличением напряжения. С помощью выражения (3) толщина барьера может быть выражена через толщину барьера при нулевом смещении на интер- фейсе:

$$
s=s_{0}(1+e U / \varphi)^{1 / 2},
$$

где $s_{0}$ - толщина барьера при нулевом смещении, $U-$ разность потенциалов на интерфейсе. Подставляя (6) в (5), получаем выражение, описывающее зависимость плотности туннельного тока через интерфейс $\mathrm{M}-$ ПП от напряжения:

$$
\begin{aligned}
j(V)= & \left(\frac{e \varphi}{2 \pi h s_{0}^{2}}\right)(1+e U / \varphi)^{-1}\{[1-e U /(2 \varphi)] \\
& \times \exp \left[-A_{0} \varphi^{1 / 2}(1-e U /(2 \varphi))^{1 / 2}(1+e U / \varphi)^{1 / 2}\right] \\
& -[1+e U /(2 \varphi)] \exp \left[-A_{0} \varphi^{1 / 2}(1+e U /(2 \varphi))^{1 / 2}\right. \\
& \left.\left.\times(1+e U / \varphi)^{1 / 2}\right]\right\},
\end{aligned}
$$

где $A_{0}=4 \pi s_{0}\left(2 m^{*}\right)^{1 / 2} / h$.

\section{5. Вольт-амперная характеристика системы симметричных встречных нелинейных контактов с низким сопротивлением}

Построение точного аналитического описания системы из нелинейных контактов с низким сопротивлением является сложной математической задачей. Однако задача существенно упрощается, если вместо точного выражения для плотности тока использовать его разложение в ряд по степеням напряжения. Такой подход, в частности, используется для описания и анализа ВАХ туннельных контактов металл-металл [11].

Мы рассматриваем систему из двух одинаковых контактов М-ПП, включенных последовательно навстречу друг другу. Из этого следует, что такая система имеет симметричную вольт-амперную характеристику, т.е.

$$
I(V)=-I(-V)
$$

Таким образом, $I(V)$ является нечетной функцией по $V$. Как известно, разложение нечетной функции содержит только нечетные степени аргумента. Таким образом, первый нелинейный член в разложении по степеням $V$ будет иметь порядок $V^{3}$, причем разложение до кубического члена будет описывать зависимость тока от напряжения с точностью о $\left(V^{4}\right)$ (,о“ малое от $V$ в четвертой степени). Учитывая слабую нелинейность туннельных контактов, наблюдаемую в эксперименте, такая точность является достаточной для практического использования. Отметим, что разложение до более высоких степеней позволяет получить описание ВАХ с любой требуемой точностью в рамках используемой аналитической модели. 


\section{1. Ток через один контакт}

Для краткости введем безразмерную переменную $u=e U / \varphi$ в выражение (7):

$$
\begin{gathered}
j(u)=k(1+u)^{-1}[(1-u / 2) \exp (-B \sqrt{(1-u / 2)(1+u)}) \\
-(1+u / 2) \exp (-B \sqrt{(1+u / 2)(1+u)})] \\
k=\frac{e \varphi}{2 \pi h s_{0}^{2}}, \quad B=A_{0} \varphi^{1 / 2}
\end{gathered}
$$

Разложение $(8)$ в степенной ряд до о $\left(u^{3}\right)$ дает

$$
\begin{aligned}
j(u)= & k e^{-B}\left[u(B / 2-1)+u^{2}\left(3 B-B^{2}\right) / 4\right. \\
& \left.-u^{3}\left(13 B+13 B^{2}-13 B^{3} / 3\right) / 64\right] .
\end{aligned}
$$

Поскольку $B / 2 \gg 1$, мы можем пренебречь единицей в первом слагаемом и упростить последнее выражение до вида

$$
\begin{aligned}
& j(u)=\frac{1}{2} k B e^{-B} \\
& \times\left[u+\frac{u^{2}}{2}(3-B)-\frac{u^{3}}{32}\left(13 B+13 B^{2}-\frac{13}{3} B^{3}\right)\right] .
\end{aligned}
$$

Чтобы получить зависимость полного тока через контакт от напряжения $U_{c}$ на нем, необходимо подставить закон зависимости напряжения от координаты (1) в (9) и провести интегрирование по $x$ от нуля до бесконечности. Учитывая, что $\int_{0}^{\infty} \exp \left(-n x / D_{0}\right) d x=D_{0} / n$, зависимость полного тока через контакт от приложенного к нему напряжения приобретает вид

$$
\begin{aligned}
I\left(u_{c}\right. & \left.=e U_{c} / \varphi\right)=L \int_{0}^{\infty} j\left(U_{c}, x\right) d x=\frac{1}{2} L D_{0} k B e^{-B} \\
& \times\left[u_{c}+\frac{u_{c}^{2}}{4}(3-B)-\frac{u_{c}^{3}}{96}\left(13 B+13 B^{2}-\frac{13}{3} B^{3}\right)\right] .
\end{aligned}
$$

\section{2. Ток во встречных контактах}

Как было сказано выше, при подаче напряжения на туннельный контакт его дифференциальное сопротивление может возрасти или уменьшиться в зависимости от направления тока. В результате при протекании одинакового тока через встречные контакты падение напряжений на них оказываются различными: на контакте, включенном в прямом направлении, напряжение будет меньше, чем на контакте, включенном в обратном направлении. При этом измеряемой экспериментально величиной является только сумма этих напряжений $V$. Обозначим напряжения на первом и втором контактах как $U_{1}(V)$ и $U_{2}(V)$ соответственно, а их разность как
$U_{1}(V)-U_{2}(V)=2 W(V)$. Тогда напряжения на каждом из контактов можно представить в виде

$$
\begin{aligned}
& U_{1}(V)=V / 2+W(V), \\
& U_{2}(V)=V / 2-W(V) .
\end{aligned}
$$

Величины $W(V), U_{1}(V)$ и $U_{2}(V)$ являются функциями полного напряжения $V$ и равны нулю при $V=0$. Заметим, что первый и второй контакты эквивалентны, поэтому при инверсии тока через систему из двух таких контактов, включенных навстречу друг другу, модули напряжений на них поменяются местами и сменят знаки на противоположные:

$$
U_{1}(V)=-U_{2}(-V) .
$$

C помощью этого соотношения легко показать, что $W(V)$ является четной функцией. Подставив (11) в (12), получим

$$
W(V)=W(-V) .
$$

Из того что $W$ является четной функцией и равна нулю при $V$, равном нулю, следует, что ее можно представить в виде разложения только по четным степеням $V$, начиная со второй. Таким образом, $W$ является величиной порядка о $(V)$. Это свойство позволяет легко выразить $W$ через величину суммарного напряжения на двух контактах, ВАХ которых заданы в виде полиномов.

Пусть ток через первый контакт описывается кубическим полиномом от $U_{1}$ вида

$$
I_{1}\left(U_{1}\right)=C_{1} U_{1}+C_{2} U_{1}^{2}+C_{3} U_{1}^{3} .
$$

Так как второй контакт эквивалентен первому, включенному в обратном направлении, зависимость протекающего в нем тока от приложенного напряжения можно выразить через те же коэффициенты, что и для первого контакта:

$$
I_{2}\left(U_{2}\right)=-I_{1}\left(-U_{2}\right)=C_{1} U_{2}-C_{2} U_{2}^{2}+C_{3} U_{2}^{3} .
$$

При последовательном включении через оба контакта протекают одинаковые токи. Приравняв правые части выражений (14a) и (14б), а также выразив напряжения на контактах через $V$ и $W$ согласно (11), получим

$$
\begin{aligned}
& C_{1}[V / 2+W(V)]+C_{2}[V / 2+W(V)]^{2}+C_{3}[V / 2+W(V)]^{3} \\
& =C_{1}[V / 2-W(V)]-C_{2}[V / 2-W(V)]^{2}+C_{3}[V / 2-W(V)]^{3} .
\end{aligned}
$$

Раскрывая скобки и пренебрегая всеми членами порядка больше $V^{3}$, находим приближение для $W$ в виде степенного ряда с точностью до о $\left(V^{3}\right)$ :

$$
W(V)=\frac{C_{2}}{C_{1}} \cdot \frac{V^{2}}{2} .
$$

Подставляя (15) в выражение для тока через первый контакт (14a), мы получаем выражение для зависимости полного тока через эквивалентные встречные контакты от суммарного напряжения на них:

$$
I(V)=C_{1}\left\{\frac{V}{2}+\left[\frac{C_{3}}{C_{1}}-2\left(\frac{C_{2}}{C_{1}}\right)^{2}\right]\left(\frac{V}{2}\right)^{3}\right\} .
$$




\section{3. Конечные выражения для вольт-амперных характеристик встречных туннельных контактов}

Подстановка коэффициентов из уравнения (10) в выражение (16) дает окончательное выражение для зависимости тока через систему из двух одинаковых туннельных контактов М-ПП, включенных навстречу друг другу:

$$
\begin{aligned}
I(V)= & L D_{0} \frac{\left(2 m^{*} \varphi\right)^{1 / 2} e^{2}}{h^{2} s_{0}} \\
& \times \exp \left(-4 \pi s_{0}\left(2 m^{*} \varphi\right)^{1 / 2} / h\right)\left[V / 2-\gamma(V / 2)^{3}\right] \\
& \gamma=\frac{23}{288} \frac{A_{0}^{2} e^{2}}{\varphi}+\frac{9}{32} \frac{A_{0}^{2} e^{2}}{\varphi^{3 / 2}}+\frac{41}{96} \frac{e^{2}}{\varphi^{2}}
\end{aligned}
$$

Для проведения расчетов выражение (17) удобно представить в практических единицах:

$$
\begin{gathered}
I=14.055 \cdot L D_{0} \frac{\sqrt{\varphi}}{s_{0}} \exp \left(-4.571 \sqrt{\varphi} \cdot s_{0}\right)\left[V / 2-\gamma(V / 2)^{3}\right] \\
\gamma=1.699 s^{2} / \varphi-1.286 s / \varphi^{3 / 2}+0.427 / \varphi
\end{gathered}
$$

Выражение (18) дает величину полного тока, выраженного в амперах, в зависимости от напряжения на них, выраженного в вольтах. Размерности других величин: ширина и длина контакта $L$ и $D_{0}-$ мкм, высота барьера $\varphi-$ эВ, толщина барьера $s_{0}-$ нм. При расчете численных коэффициентов использовано значение эффективной массы, равное 0.2 массы свободного электрона. Стоит еще раз уточнить, что в качестве напряжения $V$ в выражениях (17) и (18) используется суммарное напряжение на двух контактах, которое вычисляется путем вычитания сопротивления зазора между контактами измерительного модуля из полного напряжения на тестовом модуле $V_{\text {total, }}$ т.е. $V=\left(V_{\text {total }}-I R_{S} S / L\right)$.

\section{6. Анализ экспериментальных вольт-амперных характеристик туннельных контактов}

Выражение (18) было использовано для аппроксимации $\mathrm{BAX}$ образца 4 в интервале напряжений $U$ от 0 до 320 мВ путем минимизации среднеквадратического отклонения аппроксимирующей кривой от экспериментальных точек (рис. 4). Полученные значения высоты и ширины барьера составили $0.47 \pm 0.01$ эВ и $1.83 \pm 0.01 \mathrm{HM}$.

Основную погрешность в оценку параметров потенциального барьера по аппроксимации (17) вносит то, что функция $W(V)$ [см. выражения (11)] была приближена с точностью $\Delta V \approx(V / \varphi)^{4}$. Следовательно, относительная

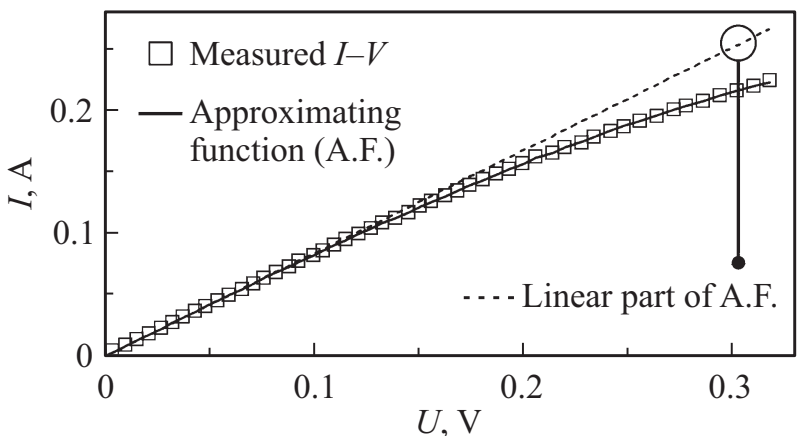

Рис. 4. Результаты аппроксимации ВАХ тестового модуля образца 4: пустые квадраты - экспериментальные точки, сплошная линия - аппроксимация, пунктирная линия линейная часть аппроксимирующей функции.

погрешность определения $\varphi$ может быть получена как интеграл

$V_{\max }^{-1} \int_{0}^{V_{\max }} \frac{\Delta V}{V} d V=V_{\max }^{-1} \int_{0}^{V_{\max }}(V / \varphi)^{3} d V=\left(V_{\max } / \varphi\right)^{4} / 4 \approx 0.05$,

где $V_{\max }$ - размах напряжения на аппроксимируемом участке на кривой, составляющий 320 мВ.

Таким образом, значения высоты и ширины потенциального барьера на границе $\mathrm{Cr}-\mathrm{GaN}: \mathrm{Si}$ составляют $\varphi=0.47 \pm 0.04$ эВ и $s_{0}=1.83 \cdot 0.11$ нм. Полученное значение высоты барьера хорошо согласуется с результатами других работ, в которых характеристики барьеров исследовались с помощью модели термоэлектронной эмиссии [5,9], и соответствует разнице между работой выхода электронов для хрома $(\approx 4.6$ эВ) и энергией сродства электронов в $\mathrm{GaN}(\approx 4.1$ эВ).

\section{7. Заключение}

В ходе исследования была разработана математическая модель для анализа нелинейных вольт-амперных характеристик планарных контактов металлполупроводник с низким удельным сопротивлением. Также в ходе работы были изготовлены и изучены контакты $\mathrm{Cr}-\mathrm{GaN}: \mathrm{Si}$ при различных концентрациях кремния в $\mathrm{GaN}$. Установлено, что при концентрации носителей $2.0 \cdot 10^{20} \mathrm{~cm}^{-3}$ транспорт электронов между металлом и $\mathrm{GaN}$ осуществляется путем туннелирования. Получено приближенное аналитическое выражение для зависимости тока через встречные диоды Шоттки, образованные одинаковыми контактами металл-полупроводник, от приложенного к ним напряжения с учетом неравномерного растекания тока по площади контактов. Путем аппроксимации экспериментальной ВАХ симметричных туннельных контактов полученным аналитическим выражением определены параметры потенциального барьеpa на границе $\mathrm{Cr}-\mathrm{GaN}$. Полученное значение высоты 
потенциального барьера составляет $0.47 \pm 0.04$ эВ и согласуется с результатами других исследований.

\section{Список литературы}

[1] Ю. Федоров. Электроника НТБ, № 2 (2011).

[2] Y. Yue, Z. Hu, J. Guo et al. IEEE Electron Dev. Lett., 33, 988 (2012).

[3] И.О. Майборода, А.А. Андреев, П.А. Перминов, Ю.В. Федоров, М.Л. Занавескин. Письма ЖТФ, 40 (11), 90 (2014).

[4] F.A. Faria, J. Guo, P. Zhao, G. Lu, P.K. Kandaswamy, M. Wistey, H. Xing, D. Jena. Appl. Phys. Lett., 101, 032109 (2012).

[5] G. Greco, F. Iucolano, F. Roccaforte. Appl. Surf. Sci., 383, 324 (2016).

[6] В.Ф. Агекян, Е.В. Борисов, Л.Е. Воробьев, Г.А. Мелентьев и др. ФТТ, 57, 768 (2015).

[7] D.K. Schroder. Semiconductor Material and Device Characterization (3rd edn) (Willey, 2006) c. 138.

[8] F. Iucolano, F. Roccaforte, A. Alberti, C. Bongiorno, S. Di Franko, V. Raineri. J. Appl. Phys., 100, 123706 (2006).

[9] M.L. Lee, J.K. Sheu, S.W. Lin. Appl. Phys. Lett., 88, 032103 (2006).

[10] Peng Zhang, Y.Y. Lau, R.M. Gilgenbach. J. Phys. D: Appl. Phys., 48, 475501 (2015).

[11] J. Simmons. J. Appl. Phys., 34, 238 (1963).

[12] H.G. Unger. Theorie der Leitungen (Friedr.Vieweg \& Sohn, 1967).

Редактор А.Н. Смирнов

\section{Tunneling current in opposite Shottky diodes formed by contacts between degenerate $\boldsymbol{n}$-type GaN and metal}

I.O. Maiboroda, J.V. Grishchenko, I.S. Ezebchenko,

I.S. Sokolov, I.A. Chernych, A.A. Andreev,

M.L. Zanaveskin

National Research Center „Kurchatov Institute“, 123182 Moscow, Russia

Abstract Nonlinear behavior of current-voltage dependences in opposite Shottky diodes formed by symmetrical contacts between metal and $n$-type degenerate $\mathrm{GaN}$ was studied for electron concentrations in $\mathrm{GaN}$ from $1.5 \cdot 10^{19} \mathrm{~cm}^{-3}$ to $2.0 \cdot 10^{20} \mathrm{~cm}^{-3}$. When electron concentration in $\mathrm{GaN}$ was $2.0 \cdot 10^{20} \mathrm{~cm}^{-3}$, the conductivity between the metal (chromium) and $\mathrm{GaN}$ was shown to be due to tunneling of electrons, and resistivity of metal-semiconductor interface was $0.05 \Omega \cdot \mathrm{mm}$. Method for characterization of potential barriers by the analysis of voltagecurrent curves of equivalent opposite contacts was developed. Nonhomogeneous distribution of current density over the contact area at low contact resistance was taken into account. Potential barrier height at chromium $-n^{+}-\mathrm{GaN}$ interface was estimated to be $0.47 \pm 0.04 \mathrm{eV}$. 